\title{
A Note on Weakly Dedekind Finite Sets
}

\author{
Pimpen Vejjajiva and Supakun Panasawatwong
}

\begin{abstract}
A set $A$ is Dedekind infinite if there is a one-to-one function from $\omega$ into $A$. A set $A$ is weakly Dedekind infinite if there is a function from $A$ onto $\omega$; otherwise $A$ is weakly Dedekind finite. For a set $M$, let $\operatorname{dfin} *(M)$ denote the set of all weakly Dedekind finite subsets of $M$. In this paper, we prove, in Zermelo-Fraenkel (ZF) set theory, that $\left|\operatorname{dfin}^{*}(M)\right|<|\mathcal{P}(M)|$ if $\operatorname{dfin}^{*}(M)$ is Dedekind infinite, whereas $\left|\operatorname{dfin}^{*}(M)\right|<|\mathcal{P}(M)|$ cannot be proved from ZF for an arbitrary $M$.
\end{abstract}

\section{Introduction and Some Basic Notions}

With the axiom of choice (AC), every set can be well ordered. As a result, the cardinality of a set can be represented by an ordinal, and so any two cardinals are comparable. Without $\mathrm{AC}$, this is no longer so. In this paper we are interested in the relation between some cardinals that can be proved from Zermelo-Fraenkel (ZF) set theory. In order to be more specific, we need the following definitions. More details of these terminologies can be found in Jech [7]. We first start by reviewing some basic notation.

Throughout this paper, we use $a, b, c, \ldots, A, B, C, \ldots$ for sets, $\alpha, \beta, \gamma, \ldots$ for ordinals, and $\mathfrak{m}, \mathfrak{n}, \mathfrak{p}, \mathfrak{q}, \ldots$ for cardinals. We write $A \approx B$ if there is a one-to-one function from $A$ onto $B, A \preceq B$ if there is a one-to-one function from $A$ into $B$, and $A \preceq * B$ if $A=\emptyset$ or there is a function from $B$ onto $A$.

Since, in this paper, we shall work in $\mathrm{ZF}$, all definitions given below are done in ZF.

Two sets have the same size or the same cardinality if they are equinumerous, that is, there is a bijection between them. We write $|A|$ for the cardinality of $A$. Then $|A|=|B| \leftrightarrow A \approx B$. Since, without AC, we cannot guarantee that a set is equinumerous to some ordinal, the definition of the cardinality of a set is given as

Received August 23, 2012; accepted October 16, 2012

2010 Mathematics Subject Classification: Primary 03E10, 03E25

Keywords: weakly Dedekind finite, Dedekind infinite, axiom of choice (C) 2014 by University of Notre Dame $\quad 10.1215 / 00294527-2688096$ 
follows:

$$
|A|=\{B \mid B \approx A \text { and } B \text { is of least rank }\} .
$$

The number $\mathfrak{m}$ is a cardinal number if $\mathfrak{m}=|M|$ for some set $M$.

For cardinals $\mathfrak{m}$ and $\mathfrak{n}$, we say that $\mathfrak{m}$ is less than or equal to $\mathfrak{n}$, written $\mathfrak{m} \leq \mathfrak{n}$, if there are $M \in \mathfrak{m}$ and $N \in \mathfrak{n}$ such that $M \preceq N$. We write $\mathfrak{m}<\mathfrak{n}$ if $\mathfrak{m} \leq \mathfrak{n}$ and $\mathfrak{m} \neq \mathfrak{n}$ and write $\mathfrak{m} \leq^{*} \mathfrak{n}$ if there are $M \in \mathfrak{m}$ and $N \in \mathfrak{n}$ such that $M \preceq^{*} N$. It is easy to see that $|M| \leq|N|$ if $M \preceq N$ and $|M| \leq^{*}|N|$ if $M \preceq^{*} N$.

Let $2^{|A|}$ denote $|\mathcal{P}(A)|$ where $\mathcal{P}(A)$ is the power set of $A$.

A set is finite if it is equinumerous to some natural number. Otherwise it is infinite.

The cardinality of an infinite well-ordered set is called an aleph. Thus an aleph is the cardinality of some ordinal: $\boldsymbol{\aleph}_{0}$ is $|\omega|$. It is easy to see that two alephs can be compared and every nonempty class of alephs has a least element.

Without AC, there may be different kinds of infinity. The following are those which will be used in this paper. More details about them can be found in Degen [1].

It is a consequence of $\mathrm{AC}$ that $\omega \preceq M$ for all infinite sets $M$ (see Howard and Rubin [6, p. 87]). We call such an infinite set Dedekind infinite. Otherwise it is Dedekind finite. We also cannot prove, in ZF, that $\omega \preceq^{*} M$ for all infinite sets $M$ (see [6, p. 87]). We call $M$ weakly Dedekind infinite if $\omega \swarrow^{*} M$. Otherwise it is weakly Dedekind finite. We can see that every finite set is weakly Dedekind finite and, since $M \preceq N$ implies $M \preceq^{*} N$ for all $M$ and $N$, every weakly Dedekind finite set is Dedekind finite.

For a set $M$ with cardinality $\mathfrak{m}$, fin $(M)$ denotes the set of all finite subsets of $M$, and let $\operatorname{fin}(\mathfrak{m})=|\operatorname{fin}(M)|, \operatorname{dfin}^{*}(M)$ denotes the set of all weakly Dedekind finite subsets of $M$, and let $\operatorname{dfin}^{*}(\mathfrak{m})=\left|\operatorname{dfin}^{*}(M)\right|$.

It has been shown that $\mathrm{ZF} \vdash \operatorname{fin}(\mathfrak{m})<2^{\mathfrak{m}}$ for all infinite $\mathfrak{m}$ (see Halbeisen and Shelah [3, Theorem 3]). Since fin $(\mathfrak{m}) \leq \operatorname{dfin}^{*}(\mathfrak{m})$, it is natural to wonder under which circumstances one can replace $\operatorname{fin}(\mathfrak{m})$ in the statement by $\operatorname{dfin}^{*}(\mathfrak{m})$. This cannot always be done, since if $X$ is weakly Dedekind finite, so are all of its subsets, and therefore $\operatorname{dfin}^{*}(X)=\mathcal{P}(X)$. In this paper, we prove, in ZF, that $\operatorname{dfin}^{*}(\mathfrak{m})<2^{\mathfrak{m}}$ if $\mathrm{dfin}^{*}(\mathfrak{m})$ is Dedekind infinite.

The following well-known theorem, whose proof can be found in any set theory textbook - for example, in Enderton [2] —is needed for the proof of Theorem 2.4.

Hartogs's theorem For every cardinal $\mathfrak{m}$, there exists a least aleph, denoted by $\aleph(\mathfrak{m})$, such that $\aleph(\mathfrak{m}) \not \leq \mathfrak{m}$.

\section{The Main Theorems}

Throughout this section, we shall work in ZF.

Lemma 2.1 For all infinite ordinals $\alpha$, fin $(\alpha) \approx \alpha$. In fact, the bijection is canonical; that is, there is a class function that to each infinite $\alpha$ assigns a bijection $\alpha \approx \operatorname{fin}(\alpha)$.

\section{Proof See [3, Corollary 3].}

The proof of [3, Theorem 3] actually gives a canonical way of assigning to each $M$, each infinite $\alpha$, and each injection of $\alpha$ into fin $(M)$ a partition $\Pi$ of $M$ with $\alpha \approx \Pi$. We begin by observing that this proof can be easily modified to show the following. 
Theorem 2.2 For all ordinals $\alpha$ and all sets $M$, if $\omega \leq \alpha \preceq \operatorname{dfin}^{*}(M)$, then $\alpha \approx \Pi$ for some partition $\Pi$ of $M$.

This follows from the argument of [3, Theorem 3], by noting that an infinite strictly decreasing sequence of sets,

$$
\operatorname{dfin}^{*}(M) \ni D_{x, \mu_{0}} \supset D_{x, \mu_{1}} \supset \cdots,
$$

immediately gives a surjection verifying $\omega \preceq^{*} D_{x, \mu_{0}}$. For the benefit of the reader, we include the details.

Proof Assume $\omega \leq \alpha \preceq \operatorname{dfin}^{*}(M)$. Then there is a one-to-one- $\alpha$ sequence $\left\langle m_{0}, m_{1}, m_{2}, \ldots, m_{\beta}, \ldots\right\rangle_{\alpha}$ of sets in $\operatorname{dfin}^{*}(M)$. Define an equivalence relation $\sim$ on $M$ by $x \sim y \Leftrightarrow \forall \beta<\alpha\left(x \in m_{\beta} \leftrightarrow y \in m_{\beta}\right)$. For any $x \in M$ and $0<\mu<\alpha$, define $D_{x, \mu}$ by

$$
D_{x, \mu}= \begin{cases}M & \text { if } x \notin m_{\beta} \text { for all } \beta<\mu, \\ \bigcap_{\iota<\mu}\left\{m_{\iota} \mid x \in m_{\iota}\right\} & \text { otherwise, }\end{cases}
$$

and define $g: M \rightarrow \mathcal{P}(\alpha)$ by $g(x)=\left\{\mu<\alpha \mid x \in m_{\mu}\right.$ and $\left.D_{x, \mu} \nsubseteq m_{\mu}\right\}$.

It is easy to see that if $x \sim y$, then $g(x)=g(y)$. The converse also holds since if $x \propto y$ and $\beta$ is the least ordinal such that $x \in m_{\beta}$ but $y \notin m_{\beta}$, then $y \in D_{y, \beta}=D_{x, \beta}$, so $D_{x, \beta} \nsubseteq m_{\beta}$, and thus $\beta \in g(x)$ but $\beta \notin g(y)$. Hence there is a one-to-one correspondence between $\{[x] \sim \mid x \in M\}$ and $\{g(x) \mid x \in M\}$.

Suppose for a contradiction that $g(x)$ is infinite for some $x \in M$. Note that for all $\mu_{1}, \mu_{2} \in g(x)$ such that $\mu_{1}<\mu_{2}, D_{x, \mu_{2}} \subset D_{x, \mu_{1}}$ because $D_{x, \mu_{2}} \subseteq m_{\mu_{1}}$ and $D_{x, \mu_{2}} \subseteq D_{x, \mu_{1}} \nsubseteq m_{\mu_{1}}$. Since $g(x)$ is an infinite set of ordinals, there is a one-toone- $\omega$ sequence $\left\langle\mu_{0}, \mu_{1}, \mu_{2}, \ldots\right\rangle_{\omega}$ of sets in $g(x)$, where $\mu_{0}<\mu_{1}<\mu_{2}<\cdots$. Then we have $m_{\mu_{0}} \supseteq D_{x, \mu_{1}} \supset D_{x, \mu_{2}} \supset \cdots$. Define $f: m_{\mu_{0}} \rightarrow \omega$ by

$$
f(y)= \begin{cases}n & \text { if } y \in D_{x, \mu_{n+1}}-D_{x, \mu_{n+2}} \text { for some } n \in \omega, \\ 0 & \text { otherwise. }\end{cases}
$$

Then $f$ is a surjection, so $\aleph_{0} \leq^{*}\left|m_{\mu_{0}}\right|$, which contradicts $m_{\mu_{0}} \in \operatorname{dfin} *(M)$.

Hence $\{g(x) \mid x \in M\} \subseteq$ fin $(\alpha)$. Since, by Lemma 2.1, fin $(\alpha) \approx \alpha$, $\{g(x) \mid x \in M\} \approx \gamma$ for some $\gamma \leq \alpha$. Since $\left\{[x]_{\sim} \mid x \in m_{\iota}\right\} \approx\left\{g(x) \mid x \in m_{\iota}\right\}$, it remains to show that $\alpha \leq \gamma$. Note first that $\gamma$ is infinite, since each set $m_{\beta}$ is union of $\sim$-classes, and there are $\alpha \geq \omega$ such sets.

Let $\eta:\{g(x) \mid x \in M\} \rightarrow \gamma$ be a bijection. Suppose for a contradiction that $\left\{\eta(g(x)) \mid x \in m_{\iota}\right\}$ is infinite for some $\iota<\alpha$. Since $\left\{[x] \sim \mid x \in m_{\iota}\right\} \approx\{\eta(g(x)) \mid$ $\left.x \in m_{\iota}\right\}$, which is an infinite set of ordinals, $\omega \preceq\left\{[x]_{\sim} \mid x \in m_{\iota}\right\}$, so there is a one-to-one- $\omega$ sequence $\left\langle\left[x_{0}\right]_{\sim},\left[x_{1}\right]_{\sim},\left[x_{2}\right]_{\sim}, \ldots\right\rangle_{\omega}$. Define a function $f^{\prime}: m_{\iota} \rightarrow \omega$ by

$$
f^{\prime}(x)= \begin{cases}n & \text { if } x \in\left[x_{n}\right]_{\sim} \\ 0 & \text { otherwise }\end{cases}
$$

Again we get a contradiction from the fact that $f^{\prime}$ is a surjection but $m_{\iota}$ is weakly Dedekind finite.

Define $h: \alpha \rightarrow \operatorname{fin}(\gamma)$ by $h(\iota)=\left\{\eta(g(x)) \mid x \in m_{\iota}\right\}$. Since $\eta$ is an injection and all $m_{\iota}$ 's are distinct, $h$ is an injection. Thus $\alpha \preceq \operatorname{fin}(\gamma) \approx \gamma$. Hence $\alpha=\gamma$, and so $\left\{[x]_{\sim} \mid x \in M\right\}$ is the partition, as desired. Finally, since the bijections $\alpha \approx \operatorname{fin}(\alpha)$ and $\gamma \approx \operatorname{fin}(\gamma)$ are canonical, so is the bijection $\{[x] \sim \mid x \in M\} \rightarrow \alpha$. 
Corollary 2.3 For all cardinals $\mathfrak{m}$, if $\aleph_{0} \leq \operatorname{dfin}^{*}(\mathfrak{m})$, then $2^{\aleph_{0}} \leq 2^{\mathfrak{m}}$.

Proof Assume $\omega \preceq \operatorname{dfin}^{*}(M)$ and $\mathfrak{m}=|M|$. By Theorem 2.2, there exists a partition $\Pi$ of $M$ such that $\omega \approx \Pi$. Let $f: \omega \rightarrow \Pi$ be a bijection. Define a function $F: \mathcal{P}(\omega) \rightarrow \mathcal{P}(M)$ by $F(x)=\bigcup f[x]$. Since $f$ is an injection, so is $F$. Hence $\mathcal{P}(\omega) \preceq \mathcal{P}(M)$, that is, $2^{\aleph_{0}} \leq 2^{\mathfrak{m}}$.

\section{Theorem 2.4 The following are equivalent:}

$$
\begin{aligned}
& \text { 1. } \aleph_{0} \leq^{*} \mathfrak{m}, \\
& \text { 2. } 2^{\aleph_{0} \leq 2^{\mathfrak{m}}}, \\
& \text { 3. } \operatorname{dfin}^{*}(\mathfrak{m})<2^{\mathfrak{m}} .
\end{aligned}
$$

Proof $(1 \Leftrightarrow 2)$ By Herrlich [5, Lemma 4.11], $\boldsymbol{\aleph}_{0} \leq^{*} \mathfrak{m} \leftrightarrow \boldsymbol{\aleph}_{0} \leq 2^{\mathfrak{m}}$ and by Halbeisen and Shelah [4, Fact 8.1], $\boldsymbol{\aleph}_{0} \leq 2^{\mathfrak{m}} \leftrightarrow 2^{\aleph_{0}} \leq 2^{\mathfrak{m}}$. This equivalence is due to Kuratowski (see Tarski [8, pp. 94-95]).

Let $\mathfrak{m}=|M|$.

$(3 \Rightarrow 1)$ If dfin $^{*}(\mathfrak{m})<2^{\mathfrak{m}}$, then there exists a set $X$ such that $\omega \preceq^{*} X \subseteq M$ and hence $\boldsymbol{\aleph}_{0} \leq{ }^{*} \mathfrak{m}$.

$(1 \Rightarrow 3)$ This follows closely to the proof of [3, Theorem 3]. It is clear that $\operatorname{dfin}^{*}(\mathfrak{m}) \leq 2^{\mathfrak{m}}$. Suppose $\aleph_{0} \leq^{*} \mathfrak{m}$ and $\operatorname{dfin}^{*}(\mathfrak{m})=2^{\mathfrak{m}}$. We will get a contradiction to Hartogs's theorem by constructing a one-to-one- $\alpha$ sequence of sets in $\operatorname{dfin}^{*}(M)$ for any $\alpha$.

Let $B: \operatorname{dfin}^{*}(M) \rightarrow \mathcal{P}(M)$ be a bijection. Let $m_{0}=B^{-1}(M)$, and for any $k \in \omega$, let $m_{k+1}=B^{-1}\left(m_{k}\right)$. Since $M \notin \operatorname{dfin}^{*}(M)$ and $B$ is an injection, the sequence $\left\langle m_{0}, m_{1}, m_{2}, \ldots\right\rangle$ is a one-to-one- $\omega$ sequence of sets in $\operatorname{dfin}^{*}(M)$.

Assume that there exists a one-to-one- $\alpha$ sequence $\left\langle m_{0}, m_{1}, m_{2}, \ldots, m_{\beta}, \ldots\right\rangle_{\alpha}$ of sets in dfin* $(M)$. We will construct an $m_{\alpha}$ by Cantor's diagonal proof that $X \prec \mathcal{P}(X)$ for any $X$, as follows.

Since $\alpha \preceq \operatorname{dfin}^{*}(M)$, by Theorem 2.2, there is a partition $\Pi$ of $M$ such that $\Pi \approx \alpha \approx\left\{m_{\iota} \mid \iota<\alpha\right\}$

Let $H:\left\{m_{\iota} \mid \iota<\alpha\right\} \rightarrow \Pi$ be a bijection. Define $h: M \rightarrow\left\{m_{\iota} \mid \iota<\alpha\right\}$ by $h(x)=m_{\iota}$ if $x \in H\left(m_{\iota}\right)$. Let $F=B \circ h$, so $F: M \rightarrow \mathcal{P}(M)$ and, as in the usual proof of Cantor's theorem, $M_{\alpha}:=\{x \in M \mid x \notin F(x)\} \notin \operatorname{ran}(F)$. Note that $h$ is a surjection and so $B\left(m_{\iota}\right) \in \operatorname{ran}(F)$ for all $\iota<\alpha$. Let $m_{\alpha}=B^{-1}\left(M_{\alpha}\right)$. Then $m_{\alpha} \notin\left\{m_{\iota} \mid \iota<\alpha\right\}$. Hence we have a one-to-one $(\alpha+1)$-sequence of sets in dfin $^{*}(M)$. Thus the proof is complete.

Since $\boldsymbol{\aleph}_{0} \leq{ }^{*} \mathfrak{m}$ for all infinite $\mathfrak{m}$ is a consequence of AC which is not provable in $\mathrm{ZF}$, Theorem 2.4 tells us that $\mathrm{dfin}^{*}(\mathfrak{m})<2^{\mathfrak{m}}$ cannot be proved from ZF for an arbitrary $\mathfrak{m}$. A condition that makes the statement provable from $\mathrm{ZF}$ is that $\mathrm{dfin}^{*}(\mathfrak{m})$ is Dedekind infinite. This is Corollary 2.5, which immediately follows by Corollary 2.3 and Theorem 2.4.

Corollary 2.5 For all cardinals $\mathfrak{m}$, if $\aleph_{0} \leq \mathrm{dfin}^{*}(\mathfrak{m})$, then $\mathrm{dfin}^{*}(\mathfrak{m})<2^{\mathfrak{m}}$.

\section{References}

[1] Degen, J. W., "Some aspects and examples of infinity notions," Mathematical Logic Quarterly, vol. 40 (1994), pp. 111-24. Zbl 0809.03036. MR 1284449. DOI 10.1002/malq.19940400116. 414 
[2] Enderton, H. B., Elements of Set Theory, Academic Press, New York, 1977. Zbl 0383.03034. MR 0439636. 414

[3] Halbeisen, L., and S. Shelah, "Consequences of arithmetic for set theory," Journal of Symbolic Logic, vol. 59 (1994), pp. 30-40. Zbl 0795.03064. MR 1264961. DOI 10.2307/2275247. 414, 415, 416

[4] Halbeisen, L., and S. Shelah, "Relations between some cardinals in the absence of the axiom of choice," Bulletin of Symbolic Logic, vol. 7 (2001), pp. 237-61. Zbl 1001.03041. MR 1839547. DOI 10.2307/2687776. 416

[5] Herrlich, H., Axiom of Choice, vol. 1876 of Lecture Notes in Mathematics, Springer, Berlin, 2006. MR 2243715. 416

[6] Howard, P., and J. E. Rubin, Consequences of the Axiom of Choice, vol. 59 of Mathematical Surveys and Monographs, American Mathematical Society, Providence, 1998. MR 1637107. 414

[7] Jech, T. J., The Axiom of Choice, vol. 75 of Studies in Logic and the Foundations of Mathematics, North-Holland, Amsterdam; American Elsevier, New York, 1973. MR 0396271. 413

[8] Tarski, A., "Sur les ensembles finis," Fundamenta Mathematicae, vol. 6 (1924), pp. 4595. 416

\title{
Acknowledgments
}

Authors' work supported by a scholarship from the Graduate School, Chulalongkorn University to commemorate the 72nd anniversary of his Majesty King Bhumibala Aduladeja. We would like to thank the anonymous referees for their suggestions and improvements.

\author{
Vejjajiva \\ Department of Mathematics and Computer Science \\ Faculty of Science, Chulalongkorn University \\ Bangkok 10330 \\ Thailand \\ pimpen.v@chula.ac.th \\ Panasawatwong \\ Department of Mathematics and Computer Science \\ Faculty of Science, Chulalongkorn University \\ Bangkok 10330 \\ Thailand \\ supakun.p@gmail.com
}

